\section{Structural and functional cardiac consequences of obstructive sleep apnea}

Obstructive sleep apnea (OSA), a sleep-related breathing disorder, can increase the risk of cardiovascular disease, leading to presentations including hypertension, arrhythmias, and congestive heart failure. Shivalkar et al. believe that the cardiac consequences of OSA could be the most important, and have investigated various cardiovascular parameters in obese patients with severe OSA who had no known history of cardiac disease.

Cardiac alterations of a structural and functional nature were clear in the patients with OSA when compared with age-matched overweight patients; OSA patients had underfunctioning left and right ventricles, dilated right ventricles, and the interventricular septum was significantly thickened $(P<0.001)$. Patients with OSA also had a higher resting heart rate than controls and were hypertensive. A significant association was seen between structural and functional cardiac parameters and apnea-hypopnea index $(P<0.004)$. Interventricular septum thickness, right ventricular dimension, and mitral annulus tissue Doppler-derived velocities were all significantly associated with a high apnea-hypopnea index $(P<0.001)$. Following 6 months of treatment with continuous positive airway pressure, OSA patients showed marked improvements in daytime sleepiness and hemodynamic, structural and functional parameters; however, improvements in diastolic function were greater in younger than in older patients.

The authors call for increased physician awareness of the cardiovascular consequences of OSA and encourage cardiovascular physicians to use methodology similar to that used in the study to evaluate patients and to help prevent severe cardiovascular consequences.

Original article Shivalkar B et al. (2006) Obstructive sleep apnea syndrome: more insights on structural and functional cardiac alterations, and the effects of treatment with continuous positive airway pressure. J Am Coll Cardiol 47: 1433-1439

\section{New role for MMP-9 in neurovascular remodeling after stroke}

Elevated expression of matrix metalloproteinases (MMPs) after stroke can promote repair and regeneration of brain tissue during delayed phases after stroke, a new study has shown. Previous studies have indicated that acute inhibition of MMPs has neuroprotective benefits, but the findings presented by Zhao et al. show that pharmacologic inhibitors might impair the recovery of brain function if given too late after stroke.

MMPs are a group of enzymes with multiple functions, including homeostasis of the extracellular matrix. With growing evidence that these enzymes can mediate blood-brain barrier damage, edema and hemorrhage in acute stroke, research efforts have been initiated to develop pharmacologic agents to suppress their activity. To compare the actions of MMP-9 in both the acute and repair phases after stroke, these researchers induced focal cerebral ischemia in male rats by transient occlusion of the right middle cerebral artery. Expression levels of MMP-9 were then assessed in sections of brain tissue obtained on days $1,3,7,10$ and 14 .

Within 1-3 days, elevated levels of MMP-9 were found in the infarct core. From days 7 to 14 , high levels of MMP-9 activity were observed in the peri-infarct cortex. MMP-9 protein also co-localized with endogenous markers of neuron and blood vessel growth. Following treatment with an MMP inhibitor at 7 days, the researchers reported suppression of neurovascular remodeling and increased ischemic brain injury.

Taken together, these findings reveal a potential role for MMPs in stroke recovery, possibly through beneficial effects on the processes of neuronal plasticity, neurogenesis and angiogenesis.

Original article Zhao B-Q et al. (2006) Role of matrix metalloproteinases in delayed cortical responses after stroke. Nat Med 12: 441-445

\section{L-arginine therapy following myocardial infarction is ineffective and possibly unsafe}

Nitric oxide deficiency is associated with endothelial dysfunction. L-arginine, a substrate for nitric oxide synthase, has been shown to improve vascular endothelial function. The VINTAGE $\mathrm{Ml}$ trial attempted to assess the effect of L-arginine therapy on vascular stiffness and left ventricular function following a first ST-segment elevation myocardial infarction. 